\title{
Alteration of Behavioral Changes and Hippocampus Galanin Expression in Chronic Unpredictable Mild Stress-Induced Depression Rats and Effect of Electroacupuncture Treatment
}

\author{
Yuping Mo, ${ }_{1}^{1}$ Haijiang Yao, ${ }^{1}$ Hongtao Song, ${ }^{2}$ Xin Wang, ${ }^{1}$ Wanshun Chen, ${ }^{1}$ Jiawula Abulizi, \\ Anping Xu, ${ }^{1}$ Yinshan Tang, ${ }^{3}$ Xiangbo Han, ${ }^{1}$ and Zhigang $\mathrm{Li}^{1}$ \\ ${ }^{1}$ School of Acupuncture, Moxibustion and Tuina, Beijing University of Chinese Medicine, No. 11 North Third Ring Road East, \\ Chaoyang, Beijing 100029, China \\ ${ }^{2}$ Department of Traditional Chinese Medicine, Inner Mongolia People's Hospital, No. 20 Zhao Wuda Road, Hohhot, \\ Inner Mongolia Autonomous Region 010017, China \\ ${ }^{3}$ Department of Traditional Chinese Medicine and Rehabilitation, The Second Affiliated Hospital of Zhejiang University School of \\ Medicine, No. 88 Jiefang Road, Hangzhou, Zhejiang 310009, China \\ Correspondence should be addressed to Zhigang Li; lizhigang620@126.com
}

Received 4 June 2014; Revised 15 September 2014; Accepted 17 September 2014; Published 30 October 2014

Academic Editor: Haruki Yamada

Copyright (C) 2014 Yuping Mo et al. This is an open access article distributed under the Creative Commons Attribution License, which permits unrestricted use, distribution, and reproduction in any medium, provided the original work is properly cited.

To explore new noninvasive treatment options for depression, this study investigated the effects of electric acupuncture (EA) for depression rat models. Depression in rats was induced by unpredictable chronic mild stress (UCMS) combined with isolation for 21 days. Eighteen male Sprague-Dawley rats were randomly assigned into three groups: control, model, and EA groups. Rats were treated by EA once daily for 21 days. The results showed that body weight and sucrose consumption were significantly increased in EA group than in the model group. The crossing numbers and rearing numbers in the open field test significantly decreased in the model group but not in the EA group. And EA treatments upregulated levels of hippocampus galanin (Gal) in UCMS rats back to relative normal levels. The present study suggested that EA had antidepressant effects on UCMS model rats. The potential antidepressant effect may be related to upregulating Gal expression in hippocampus.

\section{Introduction}

Depression is a kind of emotional disorder disease, characterized by low mood, body discomfort, and sleep disorders. Persistent state of depression, as in clinical expression, affects patients' daily life and social function. World Health Organization (WHO) forecasts that depression will be the 2nd highest disease to threaten human's health and increase the economic burden by 2020 [1].

The number of studies about acupuncture treatment for depression has increased in recent years. Electric acupuncture as a part of acupuncture treatment plays an important role gradually; effective treatments and further research are expected by many researchers and patients [2].

Neuropeptides are a kind of polypeptide which can transfer information. Hundreds of neuropeptides have been detected and found to extensively exist in nervous system and every part of the body. They take part in regulating many functions, like stress and cardiovascular activity. Many neuropeptide expressions are observed producing noticeable changes during the process of stress reaction, such as galanin (Gal), neuropeptide $\mathrm{Y}$ (NPY), vasopressin (VP), corticotrophin releasing hormone (CRH), substance $\mathrm{p}(\mathrm{SP})$, and somatostatin [3]. Neuropeptide is thought to be the upstream regulatory factor of monoamine neurotransmitter, which has great influence on neurotransmitter metabolism and function; its effect in the process of depression is the subject of much attention.

Galanin (Gal) is a kind of conservative neuropeptide. Research using ${ }^{125}$ I to mark Gal receptor showed that Gal and its binding sites were widely distributed in the whole central nervous system, and its immunocompetence showed to be 
relatively high in hippocampus, hypothalamus, and so on; another research found that Gal coexisted with acetylcholine, 5-HT, and NE such as classical neurotransmitter, and to adjust their release by hyperpolarization. The features mentioned indicate that Gal takes part in regulating many physiological functions and may be connected with cognition, emotional behavior, and other advanced physiological functions $[4,5]$.

The regulatory effect of Gal on neuroendocrine stress reactions and its involvement in anxious and depressive symptomatology have been thoroughly reviewed [6-10]. Animal behavioral studies [11-17] and a human study [18] suggest that $\mathrm{Gal}$ has a role in stress, depression like behavior, and anxiety. In addition, there is an indication from previous genetic studies on humans that the Gal system is involved in psychiatric disorders including alcoholism/addiction [19-23], panic disorder [6, 24], and chronic pain-associated depression [25]. Furthermore, recent functional studies provided the first evidence that polymorphisms in a highly conserved genetic region upstream from the $\mathrm{Gal}$ gene regulates $\mathrm{Gal}$ expression in brain areas, such as the amygdala and hypothalamus, implicated in the pathogenesis of depression $[26,27]$.

So, we think that Gal has a close relationship with depression, but there has been limited research on the effect of EA intervention to depression and its influence on Gal expression and its mechanism are not known at present.

Unpredictable chronic mild stress (UCMS) as a kind of effective depression model has been widely used in basic research and treatment selection of depression [28].

So this paper is aiming to use UCMS with isolation to establish depression model and use EA as intervention to treat depression. Detecting Gal mRNA and protein expression through real-time PCR and ELISA to explore the role of Gal in mechanism of rats' experimental depression and the effect of EA on them, the purpose is to provide new method and laboratory evidence for diagnosis and prevention of depression in clinic.

\section{Material and Methods}

2.1. Animals. A total of eighteen male Sprague-Dawley rats weighing 200 to $240 \mathrm{~g}$ were purchased from the Vital River Laboratory Animal Technology Co. Ltd. (Beijing, certificate no. SCXK(Jing)2012-0001). The animals were housed in cages at $22 \pm 2{ }^{\circ} \mathrm{C}$ and humidity of $45 \% \pm 5 \%$ under a 12 -hour light/dark cycle (lights on at 8:00 a.m., lights off at 8:00 p.m.) and received standard diet and water ad libitum. Animals were allowed to acclimatize for 3 days before the study. All experiment procedures comply with the guidelines of the "Principles of Laboratory Animal Care" formulates by the National Institute of Health and the legislation of China for the use and care of laboratory animals. The experimental protocols were approved by the Animal Experimentation Ethics Committee of Beijing University of Chinese Medicine. Efforts were made to minimize the number of animal use and the suffering of the experimental animals.

2.2. Groups and Unpredictable Chronic Mild Stress (UCMS) Model. Eighteen rats were distributed into three groups: the control group $(n=6)$, no model induction and treatment

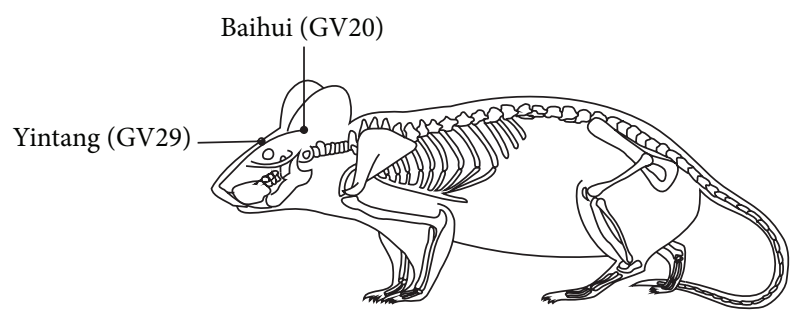

(a)

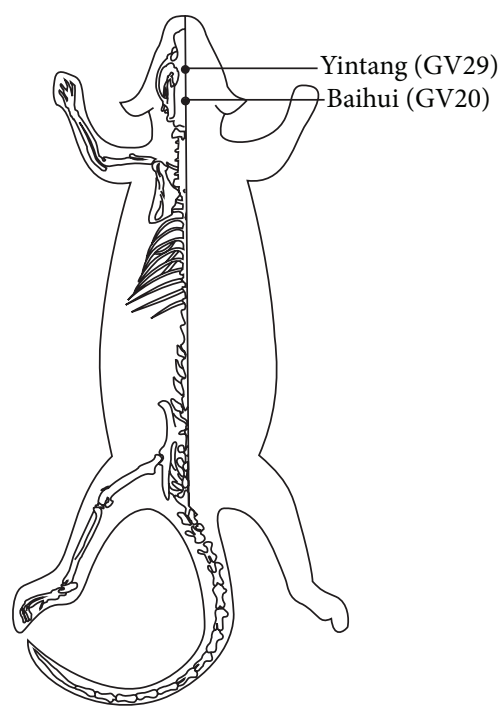

(b)

FIGURE 1: Drawings (a) and (b) show where electroacupuncture was applied.

were performed; the model group ( $n=6)$, UCMS were conducted for 21 days without treatment; the EA group $(n=$ 6), EA pretreatment was conducted daily prior to UCMS for 21 days. Every six rats in the control group were housed in one cage. However, rats in the model and EA groups were caged individually. Depression model was established by 21 days of UCMS combined with isolation. UCMS procedures contain food deprivation $(24 \mathrm{~h})$, water deprivation $(24 \mathrm{~h})$, wet bedding $(24 \mathrm{~h})$, swimming in $4^{\circ} \mathrm{C}$ ice water $(5 \mathrm{~min})$, clipping tail ( $5 \mathrm{~min}), 100 \mathrm{~V}$ electric shock $(2 \mathrm{~mA}, 5 \mathrm{~min})$, reversed light/dark cycle $(12 \mathrm{~h})$, randomly assigned 1 kind of stimulation daily, and each stimulus repeated 3 times.

2.3. EA Treatment. During acupuncture administration, rats were maintained within a cloth bag. Two points were selected: Baihui (GV20) and Yintang (GV29). GV20 is located above the apex auriculate, on the midline of the head. GV29 is located at the middle point between two eyes [29] (Figure 1). Sterilized disposable stainless steel needles $(0.30 * 25 \mathrm{~mm}$, Hua Tuo brand, manufactured by Tianjin Xinlinshuyuan Medical Devices Co., Ltd., Tianjin, China) were inserted obliquely as deep as $0.5-1 \mathrm{~cm}$ for both points. Following the insertions, electrodes were added to the handle of the needles (electric acupuncture apparatus used: LH202acupoint nerve stimulator, manufactured by Beijing Huawei Industrial Development Co., Ltd., Beijing, China). Electricity simulation parameters were $1 \mathrm{~mA}, 2 \mathrm{~Hz}$, for 20 minutes. 
2.4. Open Field Test, Sucrose Consumption, and Body Weight. The open field apparatus was constructed of black wood and measured $100 * 100 \mathrm{~cm}$ with $50 \mathrm{~cm}$ walls. White lines were drawn on the floor. The lines divided the floor. The lines divided the floor into twenty-five $20 * 20 \mathrm{~cm}$ equal squares. A single rat was gently placed in the center square to explore the arena for $5 \mathrm{~min}$, at the same time the video camera was turned on for video recording from the top of the open field apparatus. Two observers, blind to the experiment, counted the crossing numbers (defined as four paws in a square) and the rearing numbers (defined as both frontal claws uplifting from the group). The open field test was conducted at day 0 and day 22. The time of each test was fixed at 9 a.m. to 11 a.m. and was performed in a room which was with sound and light insulation and constant temperature.

The sucrose consumption test was conducted at day 0 , day 7 , day 14 , and day 21 . This method was adopted elsewhere with slight modifications [30]. The rats had free access to sucrose solution $(1 \% \mathrm{w} / \mathrm{v})$ for 3 days from the commencement of dosing for habituation. Before the test, water deprivation for $24 \mathrm{~h}$ to the rats, they were given $1 \%$ sucrose solution, totally $250 \mathrm{~g}$ with bottle. Then the rats were given a $24 \mathrm{~h}$ window sucrose test. The sucrose consumption was measured by reweighing preweighed bottles of sucrose solution. Stressors were not applied during the period of sucrose intake measurement. Control rats were housed individually during the test.

The body weight was measured on day 0 , day 7 , day 14 , and day 21 of the experiment.

2.5. Real-Time Polymerase Chain Reaction Detection of Gal $m R N A$ Expression. Rats were sacrificed at the 22nd day of the experiment for the detection of GAL mRNA expression, using the real-time polymerase chain reaction method. Rat was quickly decollated and the brain was collected on the ice plate; then hippocampal tissue was isolated and placed into $1.5 \mathrm{~mL}$ tube and stored in liquid nitrogen temporarily and then transferred to $-80^{\circ} \mathrm{C}$ refrigerator. Total RNA extraction from the specimens was performed according to the instructions of the Trizol Reagent. Reverse transcription was conducted according to the instruction of Promege Reverse Transcription System. Primers were designed and synthesized by Sangon Biotech (Shanghai) Co., Ltd., (GAL: $5^{\prime}$ CCTGAGACCACACCCACTGT- $3^{\prime}$ and $5^{\prime}$-CAGCATCAAAGCAGAGAACAAA- $3^{\prime}$; and $\beta$-actin: $5^{\prime}$-ACCGTGAAAAGATGACCCAGAT- $3^{\prime}$ and $5^{\prime}$-CCAGAGGCATACAGGGACAA $-3^{\prime}$, forward and reverse, resp.). The polymerase chain reaction was performed according to the instructions of the Applied Biosystems Step One Plus Real-Time PCR System version 2.1. In brief, the cycle conditions were $95^{\circ} \mathrm{C}$ for $10 \mathrm{~min}$ and $95^{\circ} \mathrm{C}$ for $5 \mathrm{sec}$, followed by 40 cycles at $60^{\circ} \mathrm{C}$ for $30 \mathrm{sec}, 95^{\circ} \mathrm{C}$ for $15 \mathrm{sec}, 60^{\circ} \mathrm{C}$ for $1 \mathrm{~min}$, and at $95^{\circ} \mathrm{C}$ for $15 \mathrm{sec}$. Fold-changes in gene expression were estimated using the CT comparative method normalizing to $\beta$-actin CT values and relative to control samples as follows: $\Delta \mathrm{Ct}=\mathrm{Ct} \mathrm{GAL}$ (NPY)-Ct $\beta$-actin; $\Delta \Delta \mathrm{Ct}=\Delta \mathrm{Ct}-\Delta \mathrm{Ct}$ control; fold difference $=2^{-(\Delta \Delta \mathrm{CT})}$.

2.6. Enzyme Linked Immunosorbent Assay Detection of Gal Protein Expression. Rats were sacrificed at the 22nd day of

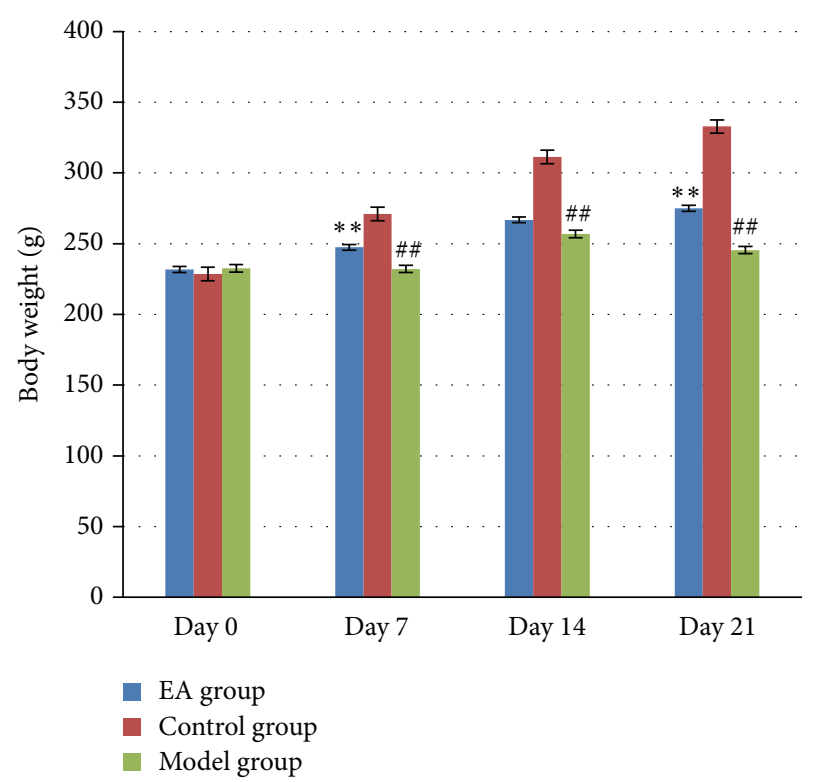

FIGURE 2: The effect of electric acupuncture (EA) on body weight at selected time points in the following groups ( $n=6$ per group): EA, control, and model. ${ }^{\# \#} P<0.01$ as compared with control group; ${ }^{* *} P<0.01$ as compared with model group.

the experiment for the detection of GAL protein expression, using the enzyme linked immunosorbent assay (ELISA) method. Rat was quickly decollated and the brain was collected on the ice plate, then hippocampal tissue was isolated and placed into $1.5 \mathrm{~mL}$ tube and stored in liquid nitrogen temporarily and then transferred to $-80^{\circ} \mathrm{C}$ refrigerator. Hippocampal was weighed and added 1XPBS (Phosphate Buffer Solution) $400 \mu \mathrm{L}$ to homogenate and then added $400 \mu \mathrm{L}$ PBS again after homogenate, placed on the ice $20 \mathrm{~min}$ (oscillation $5 \mathrm{~s}$ every $5 \mathrm{~min}$ ), centrifuged at $10000 \times \mathrm{g}$ for $5 \mathrm{~min}$ at $4^{\circ} \mathrm{C}$, and repackaged protein, and concentration of protein was detected according to the instructions of Bicinchoninic acid (BCA) (Applygen, Beijing, China). The Gal protein in rats' hippocampal was detected according to the operating steps of CUSABIO Rat galanin (GAL) ELISA Kit (Huamei, Wuhan, China) and dividing the concentration of Gal, NPY by the total concentration of protein to statistical analysis.

2.7. Statistical Analysis. Data were presented as means \pm SEM. SPSS 20.0 (SPSS Inc, Chicago, USA) was deployed for data analysis with one-way ANOVA method after the test of normal distribution and homogeneity of variance, followed by post hoc multiple comparison. Between the two groups, we used the LSD method to compare any difference. Statistical significance was set to $P<0.05$, while highly statistical significance was set to $P<0.01$.

\section{Results}

3.1. Effects of EA Treatment on Body Weight. As shown in Figure 2, the body weight increased slowly in model group 


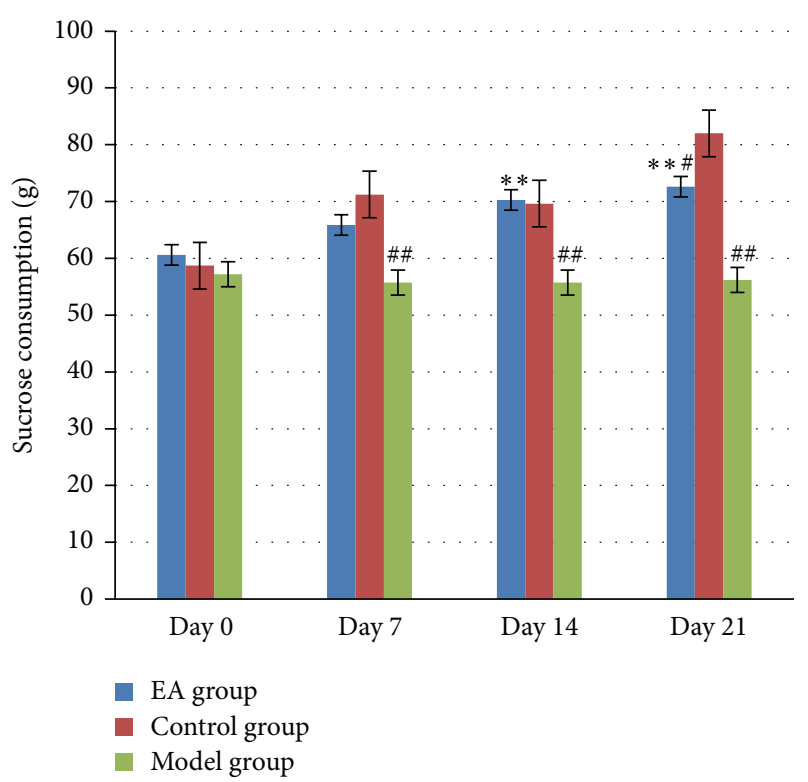

FIGURE 3: The effect of electric acupuncture (EA) on sucrose consumption at selected time points in the following groups $(n=6$ per group): EA, control, and model. ${ }^{\# \#} P<0.01$ as compared with control group; ${ }^{\#} P<0.05$ as compared with control group; ${ }^{* *} P<$ 0.01 as compared with model group.

and EA group in contrast with that in control group. Twentyone days after induction, the body weights in model group were significantly lower compared with that in control group $(P<0.01)$, whereas the body weights in EA group were significantly increased in day 7 and day 21 in comparison with that in model group $(P<0.01)$. This result indicated that UCMS has a negative influence on body weight, while EA can reverse this change which means EA has a positive effect on body weight.

\subsection{Effects of EA Treatment on Sucrose Consumption.} The sucrose consumption test is often used to measure depression-like behavior in rats by the evaluation of the hedonic state or the ability to gain pleasure [31]. As shown in Figure 3, the sucrose consumption in control group displayed an increased tendency, while those in model group and EA group increased slowly, and sucrose consumption in model group was less than that in control group with statistically significant differences $(P<0.01)$ at day 7 , day 14 , and day 21 , whereas the sucrose consumption in EA group was much more than that in model group with statistically significant differences $(P<0.01)$ at day 14 and day 21 but still less than that in control group with statistical differences $(P<0.05)$ at day 21. This result suggested that rats lost interest in sucrose which also called reward after 21 days UCMS induction, and EA can improve this situation.

3.3. Effects of EA Treatment on Open Field Test. Locomotors activity and exploratory behavior are evaluated using an open field test [32, 33]. As shown in Figures 4(a) and 4(b), 21 days after induction of the crossing numbers and rearing numbers,

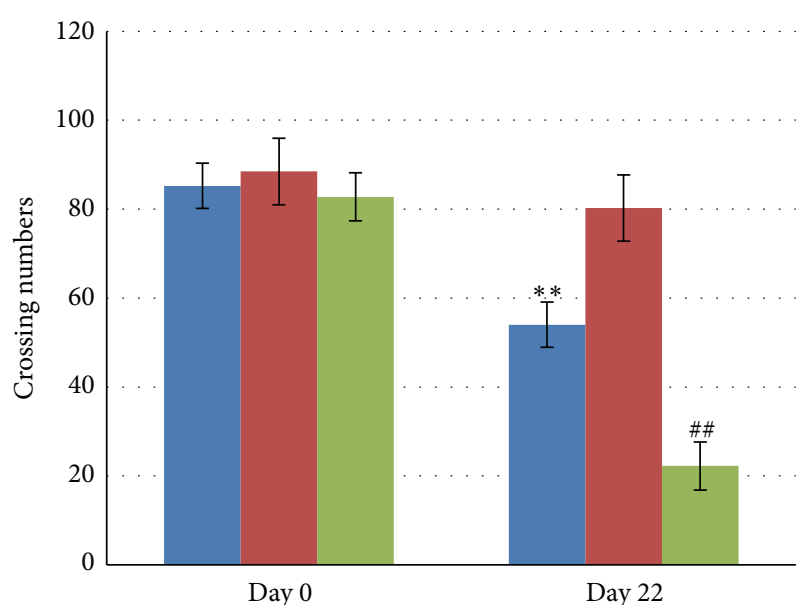

(a)

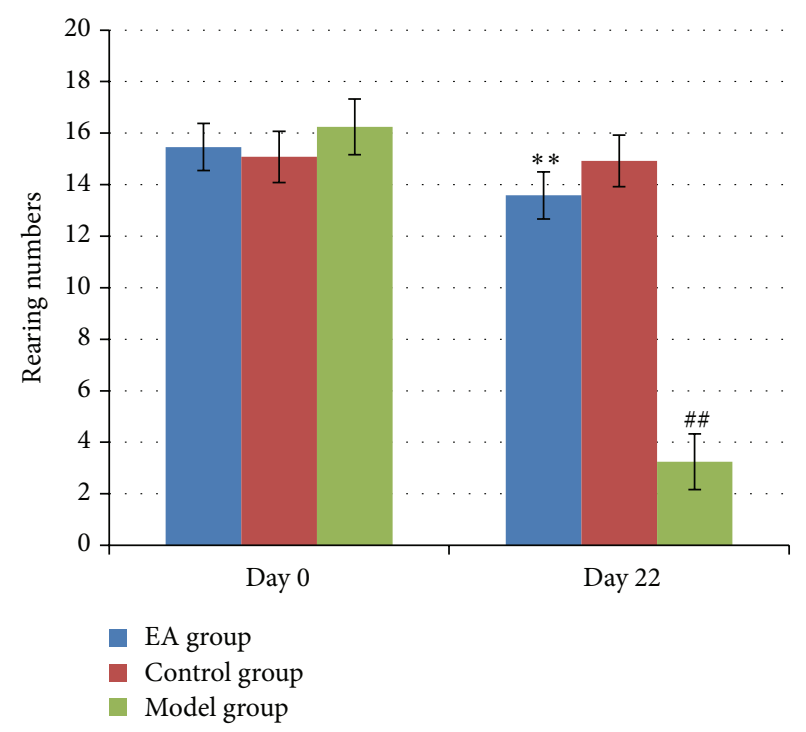

(b)

FIgURE 4: The effect of electric acupuncture (EA) on crossing numbers and rearing numbers in open field test at day 0 and day 22 in the following groups ( $n=6$ per group): EA, control, and model. (a) Crossing numbers in open field test. (b) Rearing numbers in open field test. ${ }^{\# \#} P<0.01$ as compared with control group; ${ }^{* *} P<0.01$ as compared with model group.

model group was significantly decreased in comparison with those in control group with statistically significant differences $(P<0.01)$. It indicated that UCMS model was established, while the crossing numbers and rearing numbers in EA group were increased remarkably in comparison with those in model group with statistical significance $(P<0.01)$. This result suggested that EA plays an important role in ameliorating stress-impaired exploratory and locomotor activities.

3.4. Effects of EA Treatment on GAL mRNA. As shown in Figure 5, GAL mRNA expression in hippocampus of model group decreased significantly in comparison with that in control group with statistically significant differences $(P<0.01)$, while GAL mRNA expression in hippocampus 


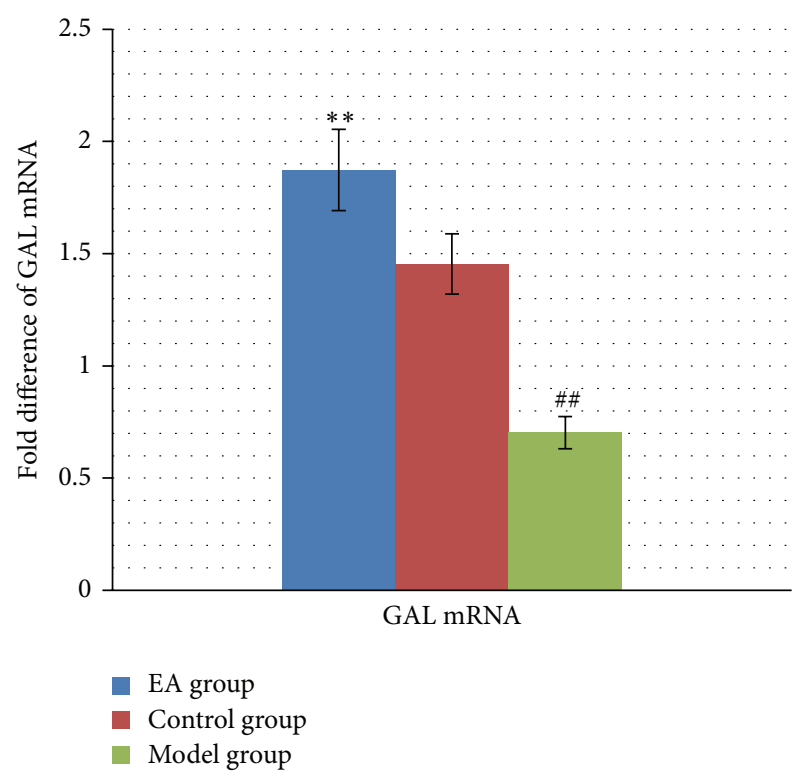

FIGURE 5: The effect of EA on GAL mRNA expression in hippocampus in the following groups ( $n=6$ per group): EA, control, and model. ${ }^{\# \#} P<0.01$, as compared with control group; ${ }^{* *} P<0.01$, as compared with model group.

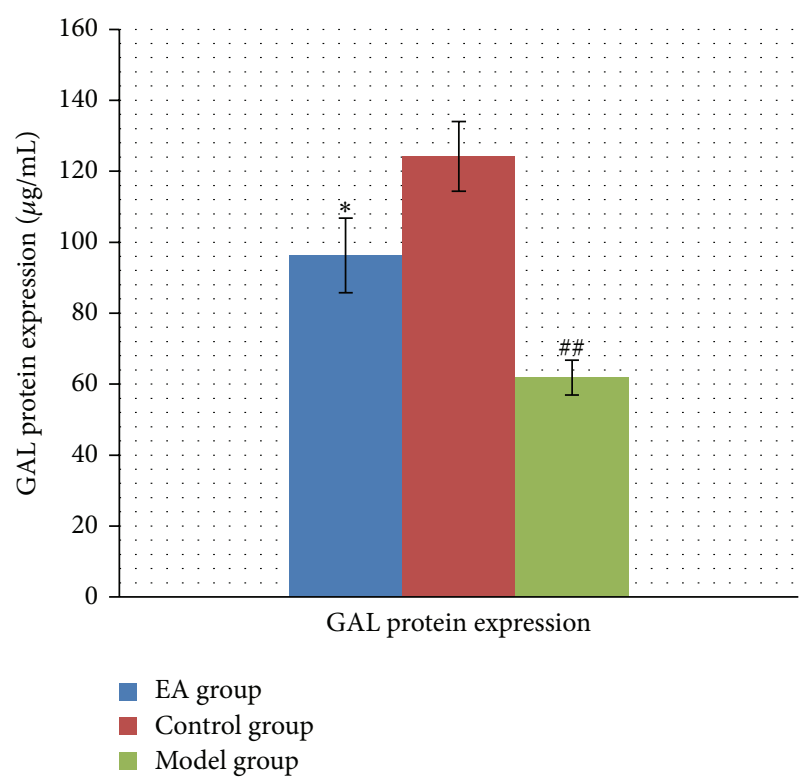

FIGURE 6: The effect of EA on GAL protein expression in hippocampus in the following groups ( $n=6$ per group): EA, control, and model. ${ }^{\# \#} P<0.01$, as compared with control group; ${ }^{*} P<0.05$, as compared with model group.

of EA group was increased remarkably in comparison with those in model group with statistical significance $(P<0.01)$. These results indicated that EA increased the expression of GAL in hippocampal tissue.

3.5. Effects of EA Treatment on GAL Protein Expression. As shown in Figure 6, GAL protein expression in hippocampus of model group decreased significantly in comparison with that in control group with statistically significant differences $(P<0.01)$, while GAL protein expression in hippocampus of EA group was increased in comparison with those in model group with statistical significance $(P<0.05)$. These results indicated that EA increased the expression of GAL in hippocampal tissue.

\section{Discussions}

The present study aims to use UCMS with isolation to establish depression model. This model, which has features of stress factor changeable and unpredictable, is closely related to the mechanism of occurrence and development of depression in human. After 21 days of induction, in model group, rats' body weight increased slowly, rewarding action weakened, and locomotor activities and explore interests reduced; while EA group could reverse this change, it indicated that the established depression model was reliable, and EA had positive effect to depression; we could come to the next step to analyze the mechanism.

The hippocampus is closely related to learning, memorizing, behavior, and emotion. It is also an important target of stress-induced injury [34].

The Gal had been studied for its role in stress and depression. Many animal experimental research showed that increasing Gal signal transduction had the effect of antidepression $[35,36]$. For example, preclinical studies had suggested that depression was associated with aberrant Gal expression, chronic stress exposure caused the reduction in $\mathrm{Gal}$ in the hippocampus of rat $[37,38]$, and giving antidepressive therapy could increase them [37, 38]. Holmes [39] has also shown that exercise exerts antidepressant effects in chronic models of depression [40], and chronic antidepressant treatment elevates Gal mRNA in the locus coeruleus (LC) similarly to exercise [41]. On the other hand, controversial studies exist. For example, it had been reported that Flinders sensitive line depression model induced an increase in Gal expression in dorsal raphe nucleus (DRN) [42] and UCMS induced an increase in Gal gene expression in amygdale and LC [42]. Shan [43] and Gao's [44] research showed that the content of Gal in experimental depression rats' hippocampus increased, but giving Gal and its receptor agonist could enhance rats' ability of independent activities and raise learning capacity. The possible explanation for the discrepancy may lie in the difference in stress category, duration, and other experimental procedures [45].

In consistency with most of these previous findings, we found that CUMS decreased Gal levels in the hippocampus. EA treatment could improve rats' behavior of depression model and increase the expression of Gal; it indicated that the increase of $\mathrm{Gal}$ content was a kind of protective regulation to depression $[43,44]$. The results were similar to that of Lu et al.s [46] research. Previous studies had demonstrated that antidepressant treatments strongly elevated Gal mRNA levels, which might be associated with induction of $\mathrm{Gal}$ synthesis and release [46]. Gal has three kinds of subtype receptors: GalR1-3, which has varying degrees of expression in central and peripheral tissue. GalR1 mRNA expressed in all of central area, expression in amygdale, and hypothalamus 
especially high [47]; GalR2 mRNA expressed relatively high in hippocampus, hypothalamus, cortex, and amygdale; GalR3 mRNA expressed high in hippocampus and hypothalamus, while expressed comparatively low in cortex and amygdale [48]. Concurrent with the increase in Gal peptide expression, an increase in GalR2-binding sites after antidepressant treatment was also found [46]. Indeed, excitatory effects of Gal, exerted probably through GalR2, on neurotransmitter release had been reported in some brain regions [46]. The use of Gal receptor antagonist, M40, underscored the findings that the increased Gal mRNA and GalR2 were relevant for the antidepressant-like effect [46]. The Gal receptor agonist, galnon, a nonselective agonist for GalR1/GalR2, was also used to underscore its acute antidepressant-like effect, which was probably similar to the effects of increased Gal release, enhanced by antidepressant treatment [46].

Subgranular zone (SGZ) of dentate gyrus in hippocampus is the place where adult mammalian's neural stem cell (NSC) regenerate; we can see high expression of $\mathrm{Gal}$ in this region. Gal combines with GalR2 which is on the membrane of NSC of SGZ, to activate PKC-ERK/MAPK signal pathway, induce NSC to mitosis, proliferation, and directional differentiation, then promote new neurons of SGZ brain region to survival, regeneration, and maturity and transfer to dentate gyrus, and then finish this neural circuitry integration [49-51]. Furthermore, activation of dorsal hippocampal GalR2 receptors facilitates cognition whereas activation of GalR1 in the ventral hippocampus impairs cognitive performance $[52,53]$, suggesting that a change in the overall action of Gal would occur when the balance between GalR1 and GalR2 is altered [46]. Therefore, we speculated that the reason which EA treatment could increase the expression of Gal might be that EA could activate GalR2 in the dorsal area of hippocampus to protect and promote the formation of cognitive function then to antidepression $[38,54]$. The combined data suggested that the galaninergic system was a putative target for EA treatment, and further researches on various animal models and clinical studies by using selective Gal receptor ligands would be needed to validate our conclusion.

According to Traditional Chinese Medicine, Baihui and Yintang are points pertaining to Governor Meridian. Brain and Governor Meridian have a direct contact on channels and collaterals. Based on the principle of Meridians pass, indications considered selecting Governor Meridian to treat mind illnesses related to brain, conforming to the Traditional Chinese Medicine saying "searching for the primary cause of disease in treatment." Meanwhile, EA treatment design of our study embodies one of the most critical theories in Traditional Chinese Medicine, the principle of "treating diseases prior to its onset" which attaches great significance on disease preventions [55].

\section{Conclusion}

In summary, the present study demonstrated that Gal in rats' hippocampus mediated the onsets of depressive symptoms after UCMS inductions. Importantly, our findings indicated that EA can significantly mitigate deficit behavioral activities elicited by UCMS through a potential mechanism of neuropeptide (Gal) modulation.

\section{Limitation}

This pilot EA study on depression has a small sample size. And the interaction among neuropeptides (Gal) and their receptors (such as GalR1-3) in different parts of brain and the effect of EA to them should be explored in the future.

\section{Conflict of Interests}

The authors declare that there is no conflict of interests regarding the publication of this paper.

\section{Authors' Contribution}

Yuping Mo and Haijiang Yao contributed equally to this paper.

\section{Acknowledgments}

This scientific work was supported by the National Natural Science Foundation (no. 81072860). The authors thank Dr. Xiaochen Yuan for his suggestions in the paper revision.

\section{References}

[1] D. J. Kupfer, "Research in affective disorders comes of age," The American Journal of Psychiatry, vol. 156, no. 2, pp. 165-167, 1999.

[2] J. C. Zhu, J. J. Wang, and M. Miao, "The research progress and overview on electroacupuncture treatment for depression," Inner Mongolia: Traditional Chinese Medicine, no. 7, pp. 117-119, 2014.

[3] S. Thakker-Varia and J. Alder, "Neuropeptides in depression: role of VGF," Behavioural Brain Research, vol. 197, no. 2, pp. 262278, 2009.

[4] Y. J. Wang, Y. T. Yang, Z. Q. Xu et al., “The research progress on Gal in the pathomechanism of depression," Journal of International Psychiatry, vol. 39, no. 3, pp. 162-165, 2012.

[5] X. P. Li, H. L. Zhou, T. Yang et al., "Influence on the expression of galanin in hippocampus of chronic unpredictable mild stressinduced depression rats," Chinese Journal of Neuroanatomy, vol. 29, no. 1, pp. 30-34, 2013.

[6] P. G. Unschuld, M. Ising, D. Roeske et al., "Gender-specific association of galanin polymorphisms with hpa-axis dysregulation, symptom severity, and antidepressant treatment response," Neuropsychopharmacology, vol. 35, no. 7, pp. 1583-1592, 2010.

[7] E. Kuteeva, T. Hokfelt, T. Wardi, and S. O. Ogren, "Galanin, galanin receptor subtypes and depression-like behaviour," Cellular and Molecular Life Sciences, vol. 65, no. 12, pp. 1854-1863, 2008.

[8] I. Mechenthaler, "Galanin and the neuroendocrine axes," Cellular and Molecular Life Sciences, vol. 65, no. 12, pp. 1826-1835, 2008.

[9] M. R. Picciotto, "Galanin and addiction," Cellular and Molecular Life Sciences, vol. 65, no. 12, pp. 1872-1879, 2008.

[10] C. C. Wrenn and J. N. Crawley, "Pharmacological evidence supporting a role for galanin in cognition and affect," Progress 
in Neuro-Psychopharmacology and Biological Psychiatry, vol. 25, no. 1, pp. 283-299, 2001.

[11] P. V. Holmes, D. C. Blanchard, R. J. Blanchard, L. S. Brady, and J. N. Crawley, "Chronic social stress increases levels of preprogalanin mRNA in the rat locus coeruleus," Pharmacology, Biochemistry and Behavior, vol. 50, no. 4, pp. 655-660, 1995.

[12] B. W. Sweerts, B. Jarrott, and A. J. Lawrence, "Expression of preprogalanin mRNA following acute and chronic restraint stress in brains of normotensive and hypertensive rats," Molecular Brain Research, vol. 69, no. 1, pp. 113-123, 1999.

[13] K. Fuxe, A. Jansson, Z. Diaz-Cabiale et al., "Galanin modulates 5-hydroxytryptamine functions: focus on galanin and galanin fragment/5-hydroxytryptamine(1A) receptor interactions in the brain," Annals of the New York Academy of Sciences, vol. 863, pp. 274-290, 1998.

[14] E. Kuteeva, T. Hökfelt, T. Wardi, and S. O. Ogren, "Galanin, galanin receptor subtypes and depression-like behaviour," EXS, vol. 102, pp. 163-181, 2010.

[15] X. Lu, L. Sharkey, and T. Bartfai, "The brain galanin receptors: targets for novel antidepressant drugs," CNS and Neurological Disorders: Drug Targets, vol. 6, no. 3, pp. 183-192, 2007.

[16] J. M. Weiss, R. W. Bonsall, M. K. Demetrikopoulos et al., "Galanin: a significant role in depression?" Annals of the New York Academy of Sciences, vol. 863, pp. 364-382, 1998.

[17] N. Kozlovsky, M. A. Matar, Z. Kaplan, J. Zohar, and H. Cohen, "The role of the galaninergic system in modulating stressrelated responses in an animal model of posttraumatic stress disorder," Biological Psychiatry, vol. 65, no. 5, pp. 383-391, 2009.

[18] H. Murck, K. Held, M. Ziegenbein, H. Künzel, F. Holsboer, and A. Steiger, "Intravenous administration of the neuropeptide galanin has fast antidepressant efficacy and affects the sleep EEG," Psychoneuroendocrinology, vol. 29, no. 9, pp. 1205-1211, 2004.

[19] I. Belfer, H. Hipp, A. Bollettino et al., "Alcoholism is associated with GALR3 but not two other galanin receptor genes," Genes, Brain and Behavior, vol. 6, no. 5, pp. 473-481, 2007.

[20] I. Belfer, H. Hipp, C. McKnight et al., "Association of galanin haplotypes with alcoholism and anxiety in two ethnically distinct populations," Molecular Psychiatry, vol. 11, no. 3, pp. 301-311, 2006.

[21] O. Levran, D. Londono, K. O'Hara et al., "Genetic susceptibility to heroin addiction: a candidate gene association study," Genes, Brain and Behavior, vol. 7, no. 7, pp. 720-729, 2008.

[22] K. J. Jackson, X. Chen, M. F. Miles, J. Harenza, and M. I. Damaj, "The neuropeptide galanin and variants in the galrl gene are associated with nicotine dependence," Neuropsychopharmacology, vol. 36, no. 11, pp. 2339-2348, 2011.

[23] A. Lori, Y. Tang, S. O'Malley et al., "The galanin receptor 1 gene associates with tobacco craving in smokers seeking cessation treatment," Neuropsychopharmacology, vol. 36, no. 7, pp. 14121420, 2011.

[24] P. G. Unschuld, M. Ising, A. Erhardt et al., "Polymorphisms in the galanin gene are associated with symptom-severity in female patients suffering from panic disorder," Journal of Affective Disorders, vol. 105, no. 1-3, pp. 177-184, 2008.

[25] M. B. Max, T. Wu, S. J. Atlas et al., "A clinical genetic method to identify mechanisms by which pain causes depression and anxiety," Molecular Pain, vol. 2, article 14, 2006.

[26] S. Davidson, M. Lear, L. Shanley et al., "Differential activity by polymorphic variants of a remote enhancer that supports galanin expression in the hypothalamus and amygdala: implications for obesity, depression and alcoholism," Neuropsychopharmacology, vol. 36, no. 11, pp. 2211-2221, 2011.
[27] J. P. Quinn, A. Warburton, P. Myers, A. L. Savage, and V. J. Bubb, "Polymorphic variation as a driver of differential neuropeptide gene expression," Neuropeptides, vol. 47, no. 6, pp. 395-400, 2013.

[28] X. Q. Li and J. Xu, "The research progress of depression animal model," Chinese Journal of Psychiatry, vol. 35, no. 3, pp. 184-186, 2002.

[29] Q. Liu, B. Li, H.-Y. Zhu, Y.-Q. Wang, J. Yu, and G.-C. Wu, "Glia atrophy in the hippocampus of chronic unpredictable stressinduced depression model rats is reversed by electroacupuncture treatment," Journal of Affective Disorders, vol. 128, no. 3, pp. 309-313, 2011.

[30] R. Muscat, M. Papp, and P. Willner, "Reversal of stressinduced anhedonia by the atypical antidepressants, fluoxetine and maprotiline," Psychopharmacology, vol. 109, no. 4, pp. 433438, 1992.

[31] E. Russo, R. Citraro, A. Davoli, L. Gallelli, E. Donato Di Paola, and G. de Sarro, "Ameliorating effects of aripiprazole on cognitive functions and depressive-like behavior in a genetic rat model of absence epilepsy and mild-depression comorbidity," Neuropharmacology, vol. 64, pp. 371-379, 2013.

[32] R.-P. Liu, J.-L. Fang, P.-J. Rong et al., "Effects of electroacupuncture at auricular concha region on the depressive status of unpredictable chronic mild stress rat models," Evidence-Based Complementary and Alternative Medicine, vol. 2013, Article ID 789674, 7 pages, 2013.

[33] L. Prut and C. Belzung, "The open field as a paradigm to measure the effects of drugs on anxiety-like behaviors: a review," European Journal of Pharmacology, vol. 463, no. 1-3, pp. 3-33, 2003.

[34] W. C. Drevets, J. L. Price, and M. L. Furey, "Brain structural and functional abnormalities in mood disorders: implications for neurocircuitry models of depression," Brain Structure and Function, vol. 213, no. 1-2, pp. 93-118, 2008.

[35] T. Bartfai, X. Lu, H. Badie-Mahdavi et al., "Galmic, a nonpeptide galanin receptor agonist, affects behaviors in seizure, pain, and forced-swim tests," Proceedings of the National Academy of Sciences of the United States of America, vol. 101, no. 28, pp. 10470-10475, 2004.

[36] E. Kuteeva, T. Wardi, L. Lundström et al., "Differential role of galanin receptors in the regulation of depression-like behavior and monoamine/stress-related genes at the cell body level," Neuropsychopharmacology, vol. 33, no. 11, pp. 2573-2585, 2008.

[37] X. D. Zheng, The study on the principle of CRH, ACTH, NPY and GAL in depression and its mechanism [Ph.D. thesis], The Second Military Medical University, Shanghai, China, 2001.

[38] X. P. Li, The expression of galanin in the hippocampus, the prefrontal cortex under chronic unpredictable stress and its neural projection [M.S. thesis], The Fourth Military Medial University, Xi'an, China, 2013.

[39] P. V. Holmes, “Trophic mechanisms for exercise-induced stress resilience: potential role of interactions between BDNF and Galanin," Front Psychiatry, vol. 5, no. 7, p. 90, 2014.

[40] H. O. Chambliss, J. D. van Hoomissen, P. V. Holmes, B. N. Bunnell, and R. K. Dishman, "Effects of chronic activity wheel running and imipramine on masculine copulatory behavior after olfactory bulbectomy," Physiology and Behavior, vol. 82, no. 4, pp. 593-600, 2004.

[41] P. V. Holmes, H. S. Yoo, and R. K. Dishman, "Voluntary exercise and clomipramine treatment elevate prepro-galanin mRNA levels in the locus coeruleus in rats," Neuroscience Letters, vol. 408, no. 1, pp. 1-4, 2006. 
[42] S. H. Christiansen, M. V. Olesen, G. Wörtwein, and D. P. D. Woldbye, "Fluoxetine reverts chronic restraint stress-induced depression-like behaviour and increases neuropeptide Y and galanin expression in mice," Behavioural Brain Research, vol. 216, no. 2, pp. 585-591, 2011.

[43] L. Shan, Study of the establishment of the depression model and galanin's effect [M.S. dissertation], East China Normal University, Shanghai, China, 2008.

[44] L. C. Gao, The involvement of neuropeptides in depression genesis and development [Ph.D. thesis], East China Normal University, Shanghai, China, 2009.

[45] J. Lu, J. Liang, J. R. Wang et al., "Acupuncture activates ERK-CREB pathway in rats exposed to chronic unpredictable mild stress," Evidence-Based Complementary and Alternative Medicine, vol. 2013, Article ID 469765, 7 pages, 2013.

[46] X. Lu, A. M. Barr, J. W. Kinney et al., "A role for galanin in antidepressant actions with a focus on the dorsal raphe nucleus," Proceedings of the National Academy of Sciences of the United States of America, vol. 102, no. 3, pp. 874-879, 2005.

[47] G. Barreda-Gómez, M. T. Giralt, and R. Rodríguez-Puertas, “G protein-coupled galanin receptor distribution in the rat central nervous system," Neuropeptides, vol. 39, no. 3, pp. 153-156, 2005.

[48] Y. M. Sui, P. H. Fang, J. Li et al., "The research progress of protective effect of Gal to nerve system," Neural Injury and Functional Reconstruction, vol. 7, no. 5, pp. 367-369, 2012.

[49] L. Wang, D. Peng, B. Xie, K. Jiang, and Y. Fang, "The extracellular signal-regulated kinase pathway may play an important role in mediating antidepressant-stimulated hippocampus neurogenesis in depression," Medical Hypotheses, vol. 79, no. 1, pp. 87-91, 2012.

[50] A. Ishii, M. Furusho, and R. Bansal, "Sustained activation of ERK1/2 MAPK in oligodendrocytes and schwann cells enhances myelin growth and stimulates oligodendrocyte progenitor expansion," Journal of Neuroscience, vol. 33, no. 1, pp. 175-186, 2013.

[51] P.-J. Shen, C.-G. Yuan, J. Ma et al., "Galanin in neuro(glio)genesis: expression of galanin and receptors by progenitor cells in vivo and in vitro and effects of galanin on neurosphere proliferation," Neuropeptides, vol. 39, no. 3, pp. 201-205, 2005.

[52] G. Fisone, C. F. Wu, S. Consolo et al., "Galanin inhibits acetylcholine release in the ventral hippocampus of the rat: histochemical, autoradiographic, in vivo, and in vitro studies," Proceedings of the National Academy of Sciences of the United States of America, vol. 84, no. 20, pp. 7339-7343, 1987.

[53] S. O. Ögren, E. Kuteeva, E. Elvander-Tottie, and T. Hökfelt, "Neuropeptides in learning and memory processes with focus on galanin," European Journal of Pharmacology, vol. 626, no. 1, pp. 9-17, 2010.

[54] T. Yoshitake, S. Yoshitake, S. Savage, E. Elvander-Tottie, S. O. Ogren, and J. Kehr, "Galanin differentially regulates acetylcholine release in ventral and dorsal hippocampus: A microdialysis study in awake rat," Neuroscience, vol. 197, pp. 172-180, 2011.

[55] T. W. Guo, Z. Guo, X. J. Yang et al., "The alterations of IL1beta, IL-6, and TGF-beta levels in hippocampal CA3 region of chronic restraint stress rats after Electroacupuncture (EA) pretreatment," Evidence-Based Complementary and Alternative Medicine, vol. 2014, Article ID 369158, 7 pages, 2014. 


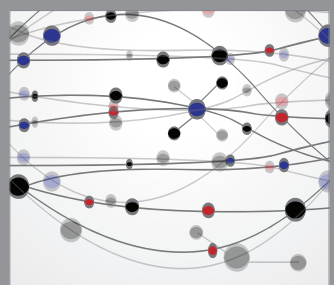

The Scientific World Journal
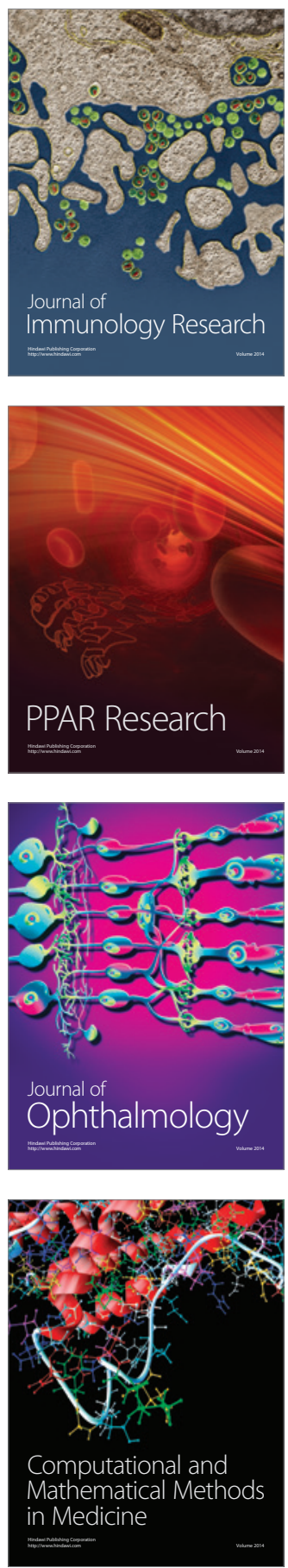

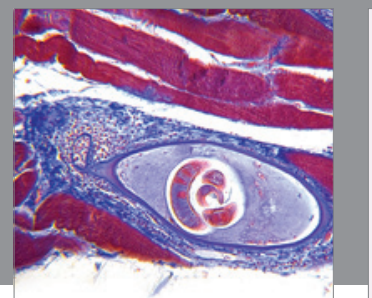

Gastroenterology

Research and Practice
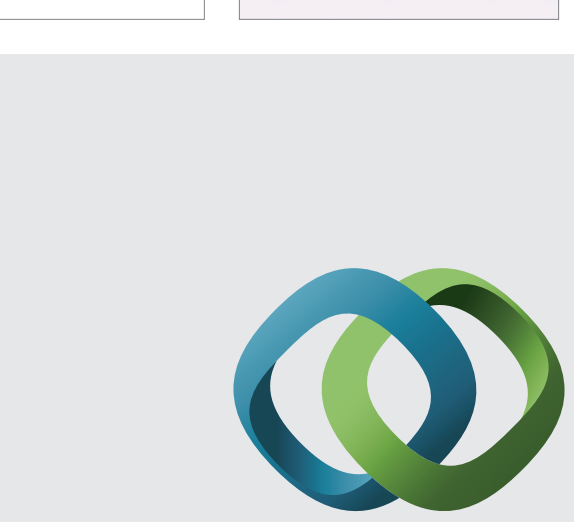

\section{Hindawi}

Submit your manuscripts at

http://www.hindawi.com
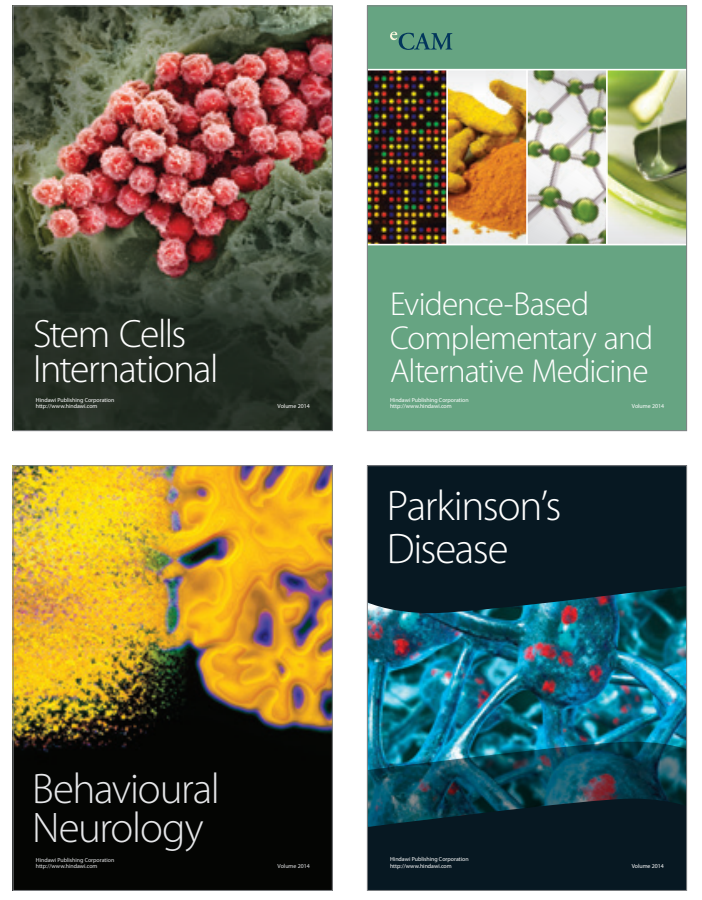
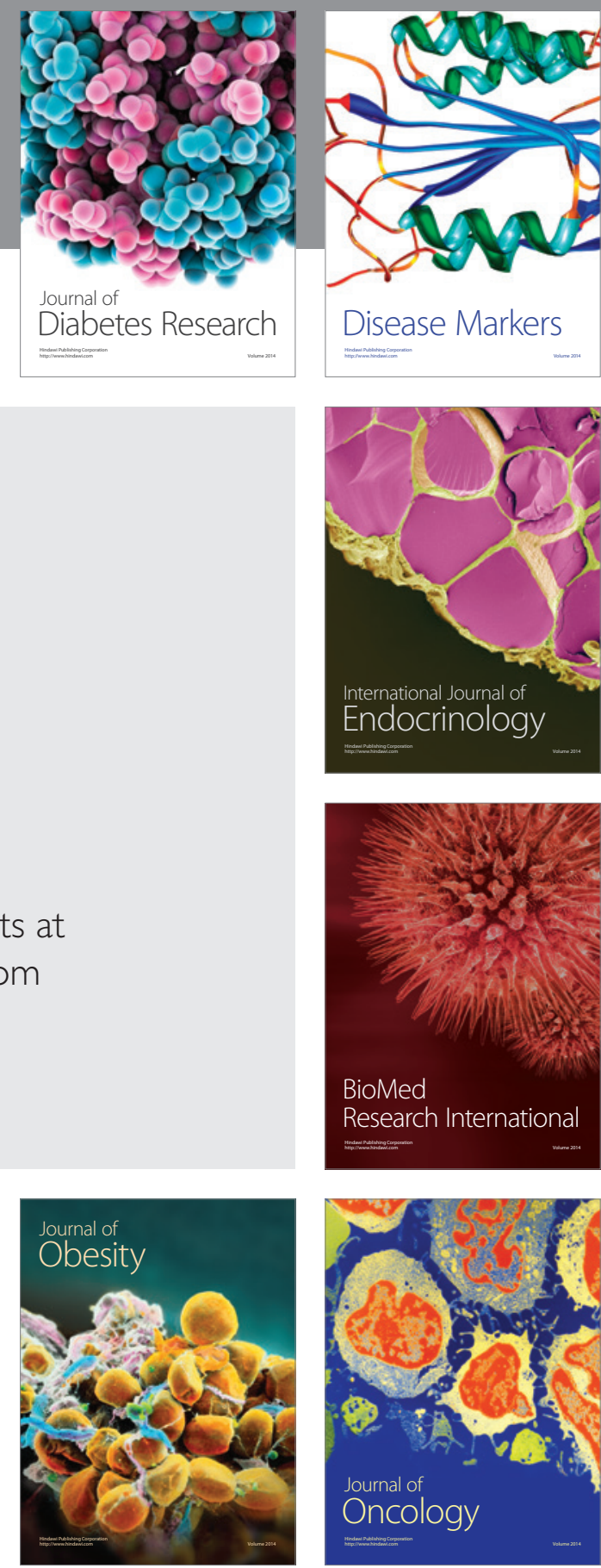

Disease Markers
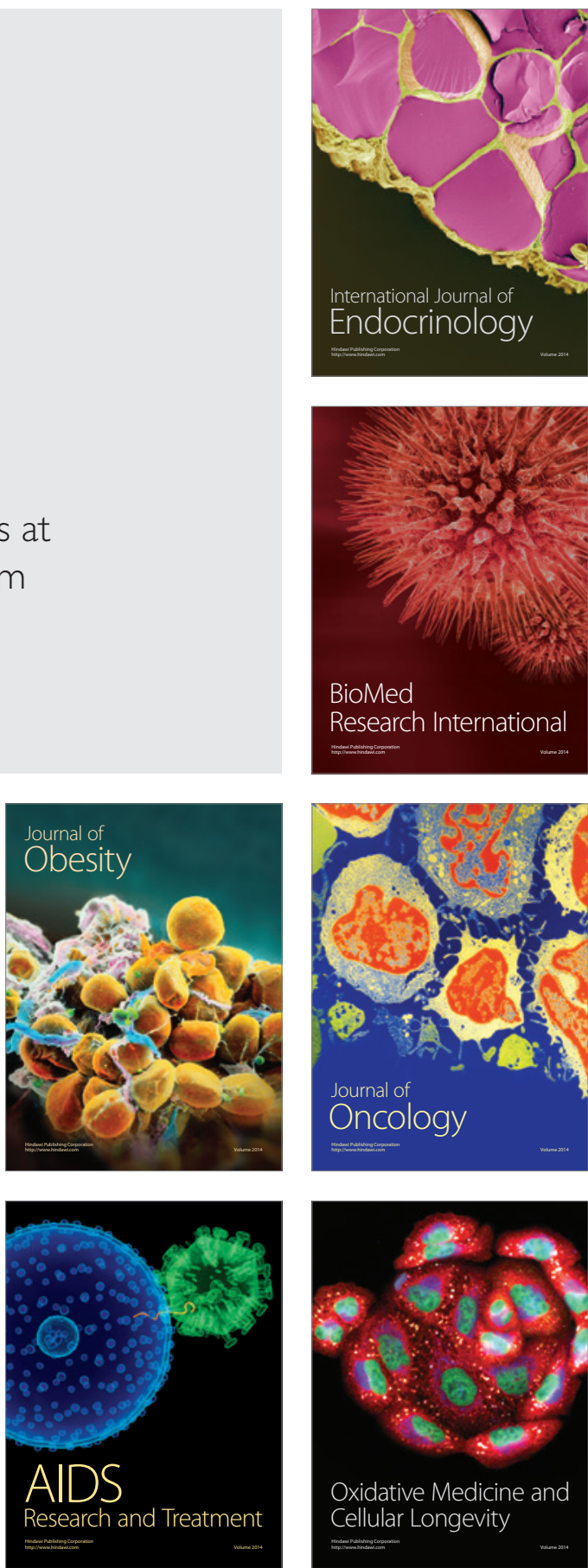